Mens

revue d'histoire intellectuelle de l'Amérique française

\title{
Mathieu Houle-Courcelles. Sur les traces de l'anarchisme au Québec (1860-1960), Montréal, Lux, 2008, 275 p.
}

\section{Francis Dupuis-Déri}

Volume 10, numéro 2, printemps 2010

URI : https://id.erudit.org/iderudit/1023310ar

DOI : https://doi.org/10.7202/1023310ar

Aller au sommaire du numéro

Éditeur(s)

Centre de recherche en civilisation canadienne-française

ISSN

1492-8647 (imprimé)

1927-9299 (numérique)

Découvrir la revue

Citer ce compte rendu

Dupuis-Déri, F. (2010). Compte rendu de [Mathieu Houle-Courcelles. Sur les traces de l'anarchisme au Québec (1860-1960), Montréal, Lux, 2008, 275 p.]

Mens, 10(2), 103-107. https://doi.org/10.7202/1023310ar d'utilisation que vous pouvez consulter en ligne.

https://apropos.erudit.org/fr/usagers/politique-dutilisation/ 
Mentionnons aussi que le livre contient des contributions de Louis Balthazar, qui nous rappelle les sympathies américaines bien connues de René Lévesque; de Guy Lachapelle, qui fait tout, sans surprise, pour nous convaincre de la modernité de son nationalisme; de Philip Resnick, sur le rapport qu'entretenait Lévesque avec le Canada anglais; de Pierre Anctil, sur son rapport avec les « communautés culturelles »; et de Pierre Nepveu, sur la littérature et René Lévesque dans un texte ésotérique qui n'a pas vraiment sa place dans un tel recueil où les contributions de bonne tenue sont plutôt la norme.

Allons à l'essentiel. On retrouve dans ce collectif un appel convaincant à rouvrir le chantier de l'histoire politique québécoise, afin de sortir de la légende dorée de la Révolution tranquille et revisiter certaines filiations inavouées dans la conscience nationale et sur le plan de nos traditions politiques. Ce bon livre dévoile surtout l'impensé progressiste du nationalisme moderne et invite les chercheurs à faire une histoire du Québec qui problématise le modernisme normalisé dans la conscience collective. Reste une conclusion, la plus importante : il faudra s'interroger sur le nationalisme québécois et ses controverses fondatrices, plus encore, sur les origines complexes du souverainisme et du Parti québécois.

\section{- Mathieu Bock-Côté \\ Département de sociologie Université du Québec à Montréal}

\section{Mathieu Houle-Courcelles. Sur les traces de l'anarchisme au Québec (1860-1960), Montréal, Lux, 2008, 275 p.}

Mathieu Houle-Courcelles nous avait habitués à la grande qualité de ses présentations de moments de l'histoire de l'anarchisme au Québec, signées sous le nom de Michel Nestor, dans les pages de 
Ruptures, la revue de la Fédération des communistes libertaires du Nord-Est - NÉFAC (dont les comités québécois se sont retirés à l'automne 2008 pour fonder l'Union communiste libertaire - UCL). Reprenant ces textes qu'il a bonifiés, Houle-Courcelles nous offre le premier livre - à notre connaissance - exclusivement consacré à l'histoire de l'anarchisme au Québec. Quelques coups de sonde avaient été lancés par des auteurs, comme Marc-André Cyr, dont l'ouvrage La presse anarchiste au Québec (1976-2001) porte sur les journaux et les revues anarchistes au Québec, et Allan Antliff, directeur d'un collectif sur les publications anarchistes au Canada (Only a Beginning: An Anarchist Anthology), ou encore Dimitrios I. Roussopoulos qui a publié un texte sur les pratiques libertaires des années 1970 et 1980 ("Perspectives anarchistes sur le Québec ", L’Arc, 1984). Le travail de Mathieu Houle-Courcelles offre toutefois une perspective beaucoup plus large que celle offerte par ces études. Il représente donc un apport unique à l'histoire du Québec.

L'auteur propose de s'attarder à trois formes d'expression de l'anarchisme, soit : 1) les individus et les groupes qui se revendiquent explicitement de l'anarchisme, « étroitement liés aux différentes vagues d'immigration ", en particulier les juifs d'Europe de l'Est au début du XXe siècle et les Espagnols à l'occasion de la Guerre civile/Révolution de 1936-1939; 2) l'anarchisme implicite, qui se manifeste "à travers des pratiques foncièrement antiautoritaires qui marquent une rupture avec les formes de pouvoir "légitimes" de la société bourgeoise (l'État, l'Église, l'armée, le régime de propriété privée) »; 3) l'anarchisme qui "se présente sous la forme d'un "spectre" agité par les milieux conservateurs et réactionnaires, mais aussi par les directions des syndicats de métier » et par la police.

Ces trois formes d'anarchisme reviennent régulièrement dans l'histoire du Québec, sans que Mathieu Houle-Courcelles insiste trop pour rappeler cette typologie au lecteur. À la recherche de ces formes, l'auteur puise nécessairement à diverses sources, qu'il manie habilement (à noter l'utilisation des citations d'époque, très bien choisies) : textes 
explicitement militants, histoires du mouvement ouvrier, du syndicalisme et de l'immigration juive, études culturelles sur des libres penseurs (Arthur Buies, par exemple) et des artistes, comme les automatistes, et divers journaux et revues (dont L'Action catholique), trouvant dans ces derniers avant tout l'expression des peurs face aux anarchistes. Pour aider à replonger dans le passé, le livre compte également près d'une vingtaine d'illustrations, entre autres, des reproductions de couvertures de brochures militantes et de journaux anarchistes et d'affiches annonçant une conférence d'Emma Goldman à Montréal ou une manifestation du $1^{\text {er }}$ Mai (1906).

Il ne manque, dans cet ouvrage, que les deux pôles historiques d'une histoire longue, soit une réfllexion sur les éléments d'anarchisme chez les autochtones d'avant la conquête européenne, alors que des historiens (Georges Sioui, par exemple) ont insisté sur leur organisation sociopolitique égalitaire et antiautoritaire. Avoir inclus une discussion des pratiques politiques et sociales autochtones aurait été en accord avec une certaine tradition historiographique anarchiste (Pierre Kropotkine et David Graeber, par exemple). C'est aussi dans cette perspective que s'inscrit la conceptualisation de l' " anarcho-indigenism " du philosophe mohawk Taiaiake Alfred, de l'Université de Victoria. Il manque aussi une présentation de l'anarchisme après les années 1950, avec des retours sur l'effet Mai 1968, la montée des groupes marxistes-léninistes dans les années 1970, du féminisme radical et de l'écologisme, puis sur l'apparition de l'altermondialisme à la fin des années 1990 (pour un aperçu, je me permets de renvoyer au texte "Fragments d'anarchisme au Québec, 2000-2006 ", de Louis-Frédéric Gaudet et Rachel Sarrasin, dans Francis Dupuis-Déri (dir.), Québec en mouvements, Montréal, Lux, 2008).

Il ne s'agit pas pour autant de faiblesses qui minent la logique du livre, puisque l'auteur a d'entrée de jeu présenté ses balises temporelles, proposant dès lors un projet qui forme un tout cohérent et qui se lit comme un véritable récit d'aventures. La plume, en effet, est habilement maniée tout au long de l'ouvrage, sans lourdeur 
stylistique ni idéologique. L'historien, suffisamment sympathique à son sujet pour en parler avec passion, ne cherche pas à en exagérer l'importance ni les succès.

Mathieu Houle-Courcelles spécifie que "l'on ne peut parler d'une "tradition" anarchiste proprement dite ", mais qu'il est possible " d'identifier une mouvance aux contours fluides qui s'est développée en de multiples tendances, dans un esprit antiautoritaire ". Pour reprendre l'expression proposée par l'auteur lui-même dans un article publié dans la revue militante Alerta! Le cri de la wawa (été 2005), l'anarchisme au Québec participerait plutôt d'un processus d' "éternel recommencement ", ce qui rend peut-être d'autant plus nécessaire d'en connaître l'histoire, puisqu'entre chaque rupture s'installe l'oubli du passé. Cette perspective de la rupture se retrouve chez d'autres historiens et théoriciens contemporains de l'anarchisme, comme le sociologue français Daniel Colson, qui explique dans Trois essais de philosophie anarchiste (2004) que l'histoire de l'anarchisme " est placée sous le signe du multiple, du disparate et du singulier, de la discontinuité et de la répétition du différent, mais dans un rapport où chacune de ses manifestations inclut, annonce et répète toutes les autres", que ce soit dans des formes aussi bien positives que négatives, comme la répression. Voilà pour les anarchistes et leurs sympathisants.

Pour les autres, le livre est d'un grand intérêt quant à l'histoire politique et culturelle du mouvement ouvrier et des idéologies, aussi bien l'anarchisme que le communisme, le syndicalisme, le féminisme et l'anticléricalisme. C'est en suivant ces pistes qu'il est possible de conclure que l'anarchisme a eu plus d'influence qu'on peut le croire en général sur l'histoire du Québec et sa société contemporaine qui tient maintenant pour acquis des principes au départ revendiqués par des anarchistes, seuls ou presque : droit pour les femmes à la contraception et à l'avortement, unions libres, accès pour tous à l'éducation, amélioration des conditions de travail, laïcisation, antifascisme, abolition de la peine de mort étatique, liberté d'assemblée et d'expression, liberté de création artistique. Une anarchiste des années 1860 qui revivrait aujourd'hui serait sans doute fort sur- 
prise de constater qu'autant d'idéaux pour lesquels elle luttait sont aujourd'hui réalisés, même si, évidemment, nous sommes loin de vivre en anarchie.

Le livre est également riche en informations sur l'histoire de Montréal, cette ville où se trouvent la plupart des scènes discutées, parfois d'une très grande précision quant à l'emplacement des librairies, des rassemblements et des restaurants ou bars où se retrouvaient les anarchistes pour discuter de leur vision du monde. Ce livre pourrait même inspirer des visites guidées du Montréal anarchiste au collectif d'animation urbaine L'Autre Montréal. On retiendra aussi l'importance des fractures linguistiques et religieuses, qui influencent ou déterminent la sphère d'influence de l'anarchisme et ses limites. Enfin, l'apport de l'anarchisme dans les luttes populaires est très bien présenté surtout en ce qui concerne les mobilisations pour des conditions de vie plus décentes -, dans l'initiative d'organiser des manifestations $\mathrm{du} 1^{\text {er }}$ Mai à Montréal, dans la mise sur pied de lieux d'éducation populaire et de comités syndicaux très revendicateurs et dans la proposition de faire éclater les normes artistiques.

- Francis Dupuis-Déri

Département de science politique

Université du Québec à Montréal

\section{Jeffrey Brison. Rockefeller, Carnegie, and Canada:American Philanthropy and the Arts and Letters in Canada, Montréal et Kingston, McGill-Queen's University Press, 2005, 261 p.}

Si les histoires de la culture canadienne du début $\mathrm{du} \mathrm{xx}^{\mathrm{e}}$ siècle n'ont pas manqué de souligner le soutien des fondations Carnegie et Rockefeller, elles ne l'avaient encore jamais considéré dans toute son ampleur. L'importante étude de Jeffrey Brison corrige cette lacune historiographique. Basée sur des recherches menées dans les archives 